\title{
Verification Results for the ISO ROSE Protocol Specified in Estelle
}

\author{
A. Jirachiefpattana, R. Lai \\ Department of Computer Science and Computer Engineering, \\ La Trobe University, \\ Victoria, Australia 3083. \\ E-mail : jira@latcs1.oz.au, lai@latcs1.oz.au
}

Existing methods of verifying Estelle specifications involve translating the specifications into another form, such as finite state machines or Petri nets. Their limitations are that they have to be based on a variant of Estelle, impose some restrictions on the specifications to be verified and do not handle the dynamic behaviours of an Estelle specification. We have developed a technique of translating a standard Estelle specification, including its dynamic behaviours, into a Numerical Petri Net specification, which can then be verified by a proven automated verification tool, PROTEAN. This paper describes the verification results for the ISO ROSE (Remote Operation Service Element) protocol specified in Estelle using this approach.

Keyword Code: C.2.2; C.2.4; F.4.3

Keywords: Computer-Communication Networks, Network Protocols, Protocol specification, testing and verification; Distributed Systems; Formal Languages

\section{INTRODUCTION}

Verification of Estelle [1, 2] specifications is still the subject of much research. Several techniques have been devised, however, these techniques have so far been based on the translation of Estelle specifications into another form, such as finite state machines, Petri nets or temporal logic formulas, for which tools of verification have already been implemented. All of the Estelle verification tools impose some restrictions on the specifications to be verified: they use a subset of Estelle or restrict the complexity of the specifications that can be verified; or the specifications need to be in a variant of Estelle, rather than standard Estelle; dynamic behaviours and exported variables of an Estelle specification are not handled.

There are a few verification tools, based on Petri nets, for Estelle specifications which have been able to verify only a verification-oriented subset of Estelle, or a variant of Estelle. Dimitrov and Petkov [3] presented an approach to automatic verification of communication protocols specified in an Estelle subset. Their approach is to limit Estelle specifications to a verification-oriented subset which could be easily translated into Petri nets. Azema et al. [4] presented a prototype for a Petri net based verifier, PIPN, which uses an underlying model for Estelle, based on Labelled Predicate/Transition Nets embedded into a logic programming environment. The main limitation of this tool is that only a static hierarchy of modules is considered. Saqui-Sannes and Courtiat [5] described 
the use and design of ESTIM, a tool for the validation of protocols specified in Estelle*, which is a variant of Estelle enhanced with rendezvous mechanism.

In our previous work [6,7], we proposed an approach to verifying standard Estelle specifications by translating them into Numerical Petri Net (NPN) specifications. In this approach, each Estelle module, consisting of a module header definition and a module body definition, is modelled independently as an indivisible NPN net which is very compact. The dynamic behaviours of an Estelle specification can be adequately modelled. However, exported variables and some Estelle statements were not included in our previous work, which is therefore not sufficient for modelling Estelle specifications in most cases, as communication entities usually use exported variables to communicate between a parent module and its associated child module; and with the information embedded in the exported variables, the parent module is able to know when to release its associated child module instance, and when to connect, disconnect, attach or detach the links. To address this limitation, we have extended the work to include exported variables, all and forone statements, and an exist expression in the NPN model. However, the Estelle priority and delay clauses, and dynamic loops are not supported using our approach.

The ISO ROSE protocol is chosen for verification because its Estelle specifications contain nearly all the Estelle keywords, like exported variables, all and forone statements, so that the viability of our approach to verifying Estelle specifications can be demonstrated. This paper describes the verification results for the ISO ROSE Estelle specifications using our techniques. The results were obtained by translating the ISO ROSE Estelle specifications into those of Numerical Petri Nets (NPNs) [8, 9], which can then be verified by a proven automated verification tool, PROTEAN [10].

\section{NUMERICAL PETRI NET (NPN)}

Numerical Petri Nets (NPNs), developed originally by Symons [11], are one of the many extensions of Petri nets. It has been found possible with NPNs to retain the basic principles, symbols and modes of operation of Petri nets, while adding a considerable amount of modelling convenience.

NPNs contain the following extensions :

- Tokens can have any finite number of attributes, each of which may be one of several different types;

- Global variables can be associated with the net;

- The transition enabling conditions refer not only to the tokens in the input places but also to the global variables;

- The firing of a transition not only removes tokens from the input places and inserts new tokens into the output places, but may change the values of the global variables.

NPNs have been successfully used in modelling some ISO protocols $[12,13]$.

\section{NPNS MODELLING ESTELLE}

The details of some of the techniques described in this section, for modelling an Estelle specification using NPNs, can be found in $[6,7]$. In this model, all module bodies and all the interaction points in the module header of any corresponding module body become 
places in an NPN, and transitions in the module body become transitions in an NPN. Tokens in NPN places indicate that the message and state belonging to the module instance will be processed by one of the enabling transitions in the module body. Transitions are fired to represent transitions being executed in the module body. The net result is the exchange of tokens or interactions from place to place, and a new marking is obtained. The mapping of Estelle components onto NPNs as dealt with in this paper is shown in Table 1.

\subsection{Translating Estelle statements}

Conditional statements in an Estelle transition block are transformed to two or more NPN transitions each of which represents a distinct control path of the original conditional statement. The transition condition of each NPN transition is modified to reflect the condition of each control path. However, we need to assume that there is no boolean expression of the conditional statement which contains variables assigned before the conditional statement.

Loop statements in an Estelle transition block are transformed by repeating the loop statement body for each value of the index variable. It is assumed here that the number of iterations can be statically determined.

Function/procedure call statements are replaced by the body of the corresponding procedure/function. In replacing the call statement, each formal parameter is symbolically replaced by an actual parameter.

With statements, which are used for record variable access, are eliminated by appending the with structure at the beginning of each record access within the statement's scope.

The generic form of all-statement, forone-statement and exist-expression is [1]:

- all domain do STATEMENT;

- forone domain suchthat BOOLEAN-EXPRESSION do STATEMENT-1

- exist domain suchthat BOOLEAN-EXPRESSION; [ otherwise STATEMENT-2 ];

The domain can be the set of either (i) all child instances of the same module-type, or (ii) vectors of values of ordinal-type(s). The details of the modelling are set out below.

All-statement It is a repetitive statement which allows iteration over an ordinal-type or over a set of module instances. In order to model this, the all-statement is eliminated by repeating the body of the STATEMENT for either each value of the ordinal type or each child instance in the domain.

Forone-statement It is a conditional statement. To model this, the forone-statement in an Estelle transition block is transformed to two or more NPN transitions each of which is for each element in the domain, or for the otherwise clause. The transition condition of each NPN transition is modified with the BOOLEAN-EXPRESSION whose variables defined in the domain are substituted with either a module instance or a value of the ordinal type in the domain. Modelling the transition representing the otherwise clause is different from the other cases in that instead of using a normal input arc, we use an inhibitor arc from the input place which holds tokens whose attributes appear in the BOOLEAN-EXPRESSION. This inhibitor arc together with the number of input tokens on the arc equal to zero means that the 


\begin{tabular}{|l|l|}
\hline Estelle & NPN \\
\hline \hline a module body & a place \\
\hline an external interaction point & a place \\
\hline an internal interaction point & a place \\
\hline a transition & a transition \\
\hline a from-clause & an enabling condition \\
\hline a provided-clause & an enabling condition \\
\hline a transition block & transition operations \\
\hline A WHEN-CLause & an in-message token \\
\hline an output-statement & an out-message token \\
\hline an exported-variable-declaration part & a place \\
\hline the module-initialization part & a transition \\
\hline a module instance and its state & a state token \\
\hline a connection status & a link token \\
\hline an attachment status & a link token \\
\hline a set of exported variables & an exported token \\
\hline an init-statement & a control token \\
\hline a release-statement & a control token \\
\hline a terminate-statement & a control token \\
\hline a connect-statement & a control token \\
\hline an attach-statement & a control token \\
\hline a disconnect-statement & a control token \\
\hline a detach-statement & a control token \\
\hline \hline
\end{tabular}

Table 1

Mapping of Estelle components onto NPNs

transition will be enabled when there are no input tokens specified on the arc in the input place.

Exist-expression Modelling the exist-expression is similar to the forone-statement except that the exist-expression does not have the do clause and the otherwise clause. However, it should be stressed that all variables defined in the domain are substituted with either a module instance or a value of the ordinal type in the domain.

\section{ISO ROSE PROTOCOL}

The ISO ROSE (Remote Operations Service Element) protocol [14, 15], which is an application service element (ASE), drives the general purpose protocol for invoking and reporting the returns of arbitrary operations. The ROSE protocol needs a transfer service to pass information in the form of ROSE APDUs between peer application-entities, and, if a connection package is involved in the contract, an association service to establish and release associations between the application-entities. Two mappings are permitted on to underlying services. ROSE may either map directly on to Association Control Service Element (ACSE) and the presentation service, or may map on to Reliable Transfer Service Element (RTSE). At present MHS is the principal user of ROSE.

Basically the ROSE service consists of establishing an application association (bind), sending a request (invoke), possibly getting a reply (result or error), and terminating the association (unbind). Each of these has service primitives associated with it. During the performance of an operation, the performance can invoke linked operations, intended to 
be performed by the invoker of the original operation. ROSE can also operate recursively; in this case the performer may initiate a further ROSE association with a third entity (a child entity) which will actually perform the operation.

Figure 1 shows the protocol model of ROSE. In this model, a connection package is involved in the contract, so the ACSE service is used to establish and release associations between the application-entities. In addition, this model is concatenation-prohibited; that is, each TRANSFER request corresponds to a single P-DATA request.

\section{ESTELLE SPECIFICATION OF ROSE}

Figure 1 shows the environment and the module structure in which the Estelle specification of ROSE protocol has been considered. The USER module represents an application above ROSE. Since ACSE is the association realisation which provides services for the establishment and release of associations, the transfer realisation is based on the use of P-DATA to transfer the APDUs. The PS module represents the presentation service provider which provides transfer services to the ROPM module. The ROPM module specifies the ROSE protocol machine which consists of four modules: (i) BROPM (ROPM providing basic ROSE services), (ii) AROPM (ROPM providing association establishment and release), (iii) TROPM (ROPM providing information transfer) and (iv) ACPM (Association Control Protocol Machine). These four modules are created and released dynamically.

The Estelle specification has about 3500 lines, and contains more than 100 transitions. Thus, it is not possible to list in this paper the whole Estelle specification. Instead, the association establishment described in Estelle can be found in Appendix A. The following features were not included in the Estelle specification: linked operations, recursive operations and concatenation-permitted transfer services.

\section{TRANSLATING ROSE INTO NPN}

Each Estelle module is translated independently into an indivisible NPN net and later all of these nets are integrated. As shown in Appendix A, the Estelle specification is composed of 4 sibling modules, BROPM, AROPM, TROPM and ACPM, all of which are under one parent module, ROPM. Figure 5 gives the global NPN net of the Estelle specification.

In the NPN net representing the BROPM module, there are 5 NPN places: BROPM_body representing the module body identifier; BROPM_BROSE, BROPM_ABROSE and BROPM _TBROSE representing the associated interaction point identifiers BROSE, ABROSE and TBROSE respectively; and BROPM_EXPORT representing the associated exportedvariable-declaration part. Six Estelle transitions in the BROPM module, ESTreq, RO_BINDind, RO_BINDcnfA, RO_BINDcnfE, ESTrspA and ESTrspE, are translated to be six NPN transitions; and there is one additional NPN transition, InitBROPM, for the initialization part of the module.

In the NPN net representing the AROPM module, there are also 5 NPN places: AROPM_body representing the module body identifier; AROPM_ABROSE, AROPM_ AROSE and AROPM_ATROSE representing the associated interaction point identifiers ABROSE, AROSE and ATROSE respectively; and AROPM EXPORT representing the 


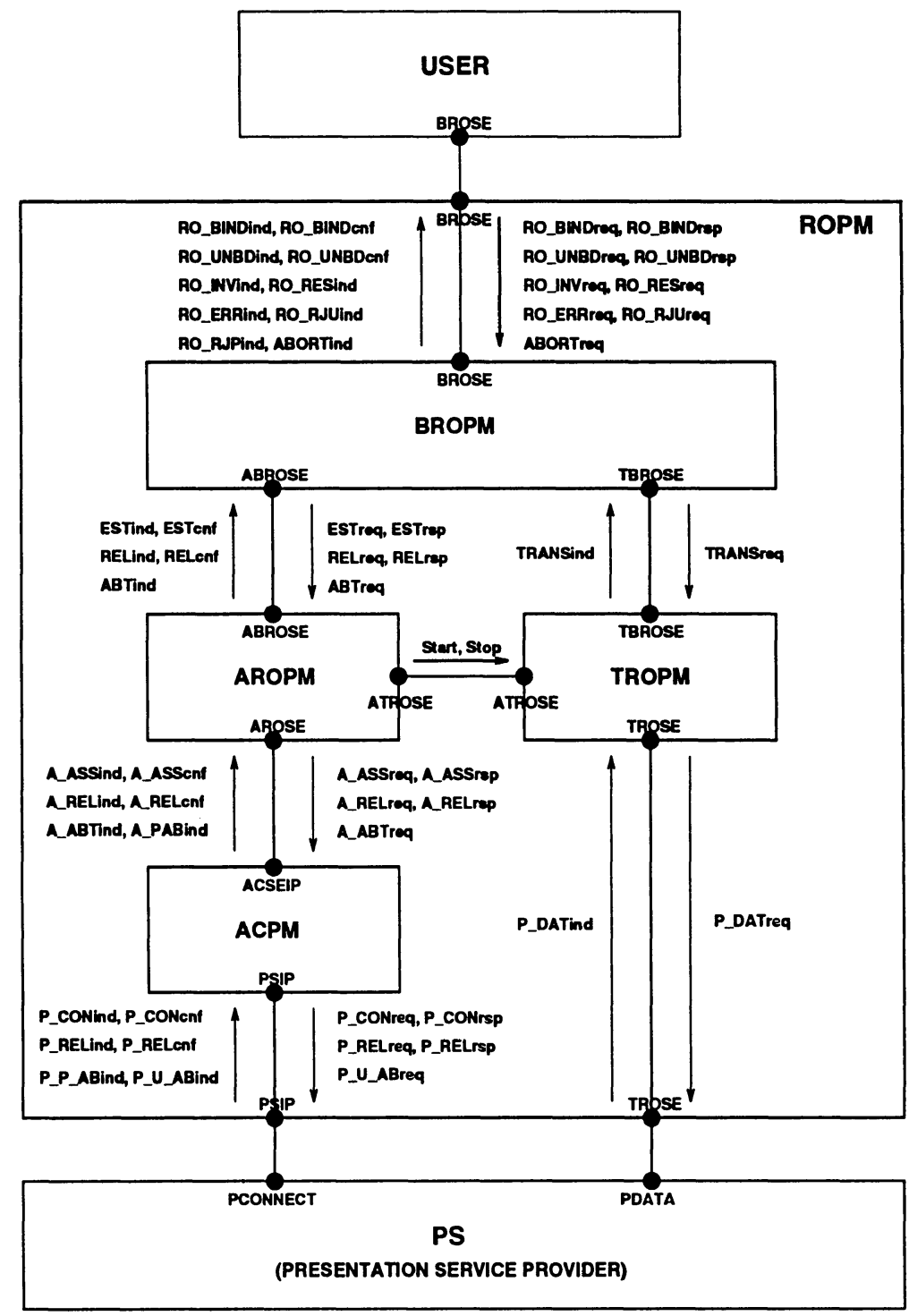

ROPM - Remote Operations Protocol Machine BROPM - ROPM providing basic ROSE services TROPM - ROPM providing information transfer
AROPM - ROPM providing association establishment and release ACPM - Association Control Protocol Machine

Figure 1. Protocol Model of ISO ROSE 
associated exported-variable-declaration part. Six Estelle transitions in the AROPM module, A_ASSreq, ESTind, A_ASSrspA, A_ASSrspE, ESTcnfA and ESTcnfE, are translated to be six NPN transitions; and there is one additional NPN transition, InitAROPM, for the initialization part of the module.

In the NPN net representing the TROPM module, there are also 5 NPN places: TROPM_body representing the module body identifier; TROPM_TBROSE, TROPM_ TROSE and TROPM_ATROSE representing the associated interaction point identifiers TBROSE, TROSE and ATROSE respectively; and TROPM_EXPORT representing the associated exported-variable-declaration part. Four Estelle transitions in the TROPM module, Start, PDATreq, TRANSind and Stop, are translated to be four NPN transitions; and there is one additional NPN transition, InitTROPM, for the initialization part of the module.

In the NPN net representing the ACPM module, there are 4 NPN places: ACPM body representing the module body identifier; ACPM_ACSEIP and ACPM_PSIP representing the associated interaction point identifiers ACSEIP and PSIP respectively; and ACPM_ EXPORT representing the associated exported-variable-declaration part. Six Estelle transitions in the ACPM module, P_CONreq, A_ASSind, P_CONrspA, P_CONrspE, A_ASScnfA and A_ASScnfE, are translated to be six NPN transitions; and there is one additional NPN transition, InitACPM, for the initialization part of the module.

In the last NPN net representing the ROPM module, which is the parent of the modules mentioned above, there are 4 NPN places: ROPM_body representing the module body identifier; and ROPM_BROSE, ROPM_TROSE and ROPM_PSIP representing the associated interaction point identifiers BROSE, TROSE and PSIP respectively. Three Estelle transitions in the ROPM module, CreateChildrenA, CreateChildrenB and ReleaseROPMChildren, are translated to be three NPN transitions; and there is one additional NPN transition, InitROPM, for the initialization part.

In order to integrate all of these NPN nets, there are:

- one input arc from the NPN place ROPM_BROSE to the NPN transition CreateChildrenA; ChildrenB;

- one input arc from the NPN place ROPM_PSIP to the NPN transition Create-

- four input arcs from the NPN places BROPM_EXPORT, AROPMEXPORT, TROPM_EXPORT and ACPM_EXPORT to the NPN transition ReleaseROPMChildren;

- eleven output arcs from the transition CreateChildrenA and also from the transition CreateChildrenB to eleven NPN places: BROPM_body, BROPM_ABROSE, BROPM _TBROSE, AROPM_body, AROPM_AROSE, AROPM_ATROSE, ACPM_body, TROPM_ body, ROPMBROSE, ROPM_TROSE and ROPM_PSIP;

- eight output arcs from the transition ReleaseROPMChildren to eight NPN places: BROPM_body, BROPM_EXPORT, AROPM_body, AROPM_EXPORT, TROPM_body, TROPM_EXPORT, ACPM_body and ACPM_EXPORT;

- three NPN transitions: Link_ROPM_BROPM, ROPM_BROPM and BROPM_ROPM, to integrate the net representing the ROPM module and the net representing the BROPM module;

- three NPN transitions: Link_BROPM_AROPM, BROPM_AROPM and AROPM_ $\mathrm{BROPM}$, to integrate the net representing the BROPM module and the net representing 
the AROPM module;

- three NPN transitions: Link_BROPM_TROPM, BROPM_TROPM and TROPM_ BROPM, to integrate the net representing the BROPM module and the net representing the TROPM module;

- three NPN transitions: Link_AROPM_ACPM, AROPM_ACPM and ACPM_

AROPM, to integrate the net representing the AROPM module and the net representing the ACPM module;

- three NPN transitions: Link_AROPM_TROPM, AROPM_TROPM and TROPM_ AROPM, to integrate the net representing the AROPM module and the net representing the TROPM module;

- three NPN transitions: Link_ROPM_TROPM, ROPM_TROPM and TROPM_ ROPM, to integrate the net representing the ROPM module and the net representing the TROPM module;

- three NPN transitions: Link_ROPM_ACPM, ROPM_ACPM and ACPM_ROPM, to integrate the net representing the ROPM module and the net representing the ACPM module.

\section{VERIFICATION RESULTS}

After translating the Estelle specification into NPNs, the behaviour of the protocol is analysed using an automated verification tool, PROTEAN (PROTocol Emulation and ANalysis) [10], developed by Telecom Australia. PROTEAN is a proven verification tool for verifying complex application layer protocols [16]. The verification technique employed by PROTEAN is Reachability analysis [17]. The methods of using PROTEAN for verifying protocols are described in [18]. The verification exercises revealed that the protocol functions without any deadlock or livelock in all the normal cases when collision does not occur. However, in the case of an association-release with collision but without any operations in the "unbind pending" state, 10 deadlocks and no livelock have been uncovered.

The details of each deadlock are set out below:

\subsection{DEADLOCKS 1, 2 AND 3}

Figure 2 shows a sequence of service primitives leading to deadlock 1 . This happens when the association-responder ROSE user, UB, issues a RO_UNBDreq primitive, and simultaneously the association-responder BROPM issues a RO_UNBDind primitive after receiving a RELind primitive. Then, UB changes to the "local unbind pending" state, whereas the association-responder BROPM changes to the "remote unbind pending" state in which it does not accept any service primitives from UB other than a RO_UNBDrsp primitive. Once UB receives the RO_UNBDind primitive, it understands that there is a release collision, but the association-responder BROPM does not. So, UB will not issue a RO_UNBDrsp primitive, but will change from its current state to the "associationresponder-unbind-collision" state in which it expects to receive a RO_UNBDcnf primitive. As a result, a deadlock occurs.

The causes of deadlocks 2 and 3 are similar to that of deadlock 1, except that deadlock 2 occurs between the association-responder AROPM and its service-user and deadlock 3 occurs between the association-responder ACPM and its service-user. 


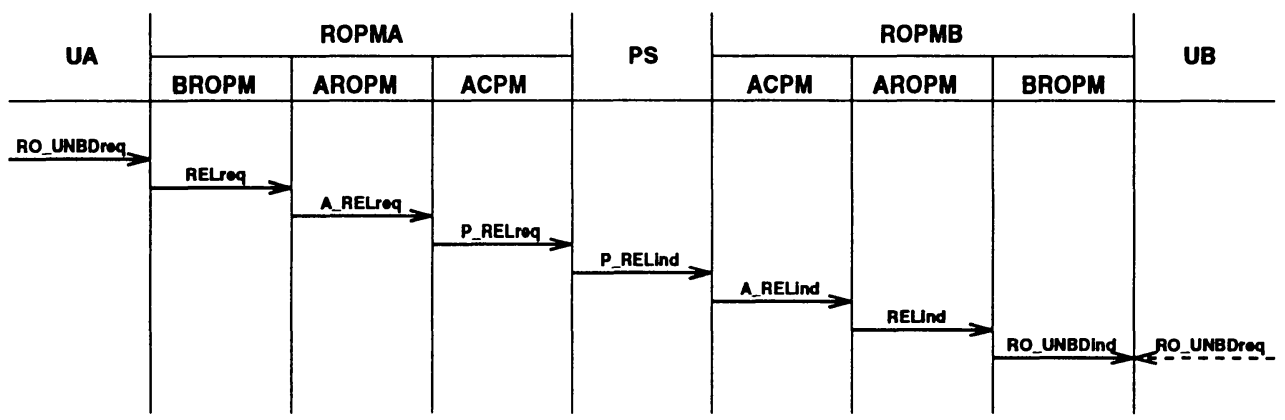

Figure 2. A sequence of service primitives causing Deadlock 1

\subsection{DEADLOCKS 4, 5 AND 6}

Figure 3 shows a sequence of service primitives leading to deadlock 4 . This happens when the association-initiator ROSE user, UA, issues a RO_UNBDreq primitive, and simultaneously the association-initiator BROPM issues a RO_UNBDind primitive. Then, UA changes to the "local unbind pending" state, whereas the association-initiator BROPM changes to the "remote unbind pending" state in which it does not accept any service primitives from UA other than a RO_UNBDrsp primitive. Once UA receives the RO_UNBDind primitive, it understands that there is a release collision, but the association-initiator BROPM does not. So, UA will change from its current state to the "association-initiatorunbind-collision" state in which it will issue a RO_UNBDrsp primitive, and then expect to receive a RO_UNBDcnf primitive. After the association-initiator BROPM receives the RO_UNBDrsp primitive from its service-user, it issues a RELrsp primitive to the association-initiator AROPM, and then changes from its current state to the "unbound" state in which the association is released. But the association-initiator ROSE user, UA, is still waiting for a RO_UNBDcnf primitive. As a result, a deadlock occurs.

The causes of deadlocks 5 and 6 are similar to that of deadlock 4, except that deadlock 5 occurs between the association-initiator AROPM and its service-user and deadlock 6 occurs between the association-initiator ACPM and its service-user.

\subsection{DEADLOCKS 7, 8, 9 AND 10}

Figure 4 shows a sequence of service primitives leading to deadlock 7 . This happens when both the association-initiator ROSE user, UA, and the association-responder ROSE user, UB, issue a RO_UNBDreq primitive, and only the association-initiator side recognizes that there is a release collision while the association-responder side does not. The reason is that the P_RELind primitive issued by the association-initiator side is lost, or is received by the association-responder ACPM after the P_RELcnf primitive which was issued by the association-initiator side in response to the release request from the association-responder side. That is, the association-initiator side understands that there is a release collision, and is waiting for an unbind confirm from the association-responder side; whereas the association-responder side understands that there is no collision, and 


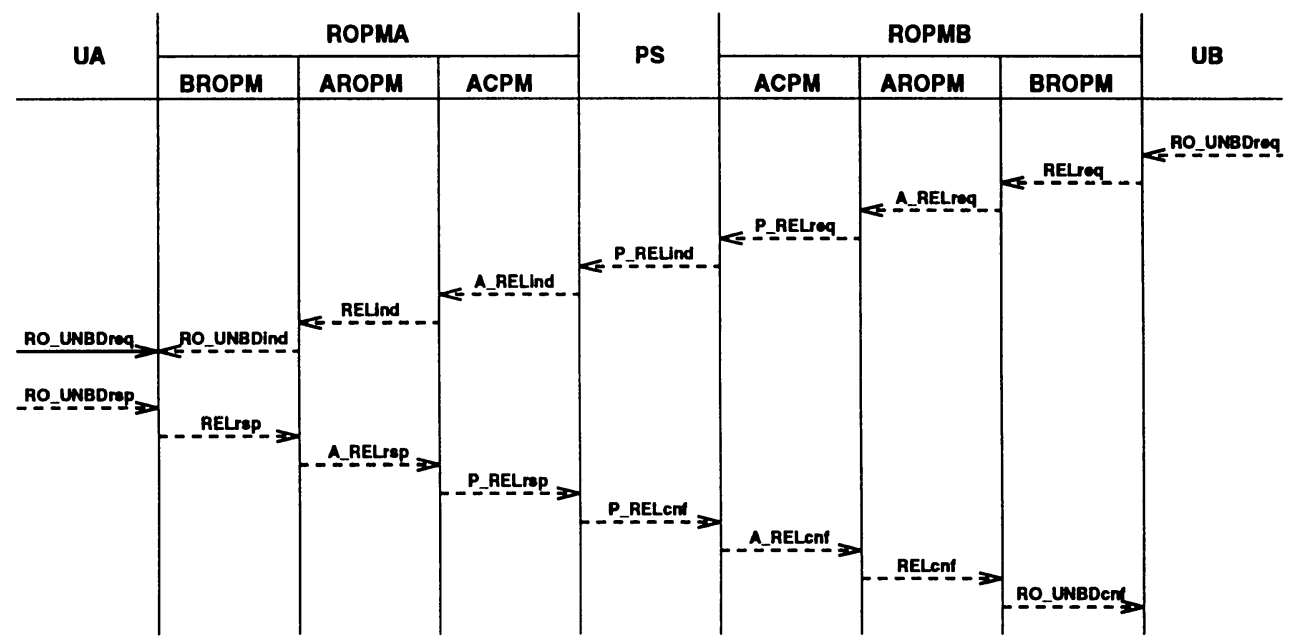

Figure 3. A sequence of service primitives causing Deadlock 4

then changes from its current state to the "unbound" state after receiving an unbind confirm from the association-initiator side. Consequently, a deadlock occurs.

The causes of deadlocks 8,9 and 10 are similar to that of deadlock 7 ; for deadlock 8 , the difference is that the A_RELind primitive is lost or is received by the associationresponder AROPM after the A_RELcnf primitive; for deadlock 9, it is that the RELind primitive is lost or is received by the association-responder BROPM after the RELcnf primitive; and for deadlock 10, it is that the RO_UNBDind primitive is lost or is received by the association-responder ROSE user, UB, after the RO_UNBDcnf primitive.

\section{CONCLUSIONS}

In this paper, we have described the verification results for the ISO ROSE Estelle specifications. They are obtained by translating the Estelle specifications into Numerical Petri Net specifications, which are subsequently verified by using a proven automated tool, PROTEAN. While no livelock was identified, we uncovered ten deadlocks caused by collision problems.

The present translation is done manually; this is the most time-consuming part. We are developing a tool, ESVN (Estelle Verification Using NPNs), for the automatic translation of Estelle specifications into NPNs. ESVN will generate intermediate codes that allow verification to be performed by PROTEAN. Any error detected during the verification process will be reported by ESVN in Estelle format. Once the development of the software is completed, verification of Estelle specifications will then be automatic as the user will not need to know anything about the translation; the user will also be able to understand the errors detected more readily as they will be described in Estelle format. Until then, 


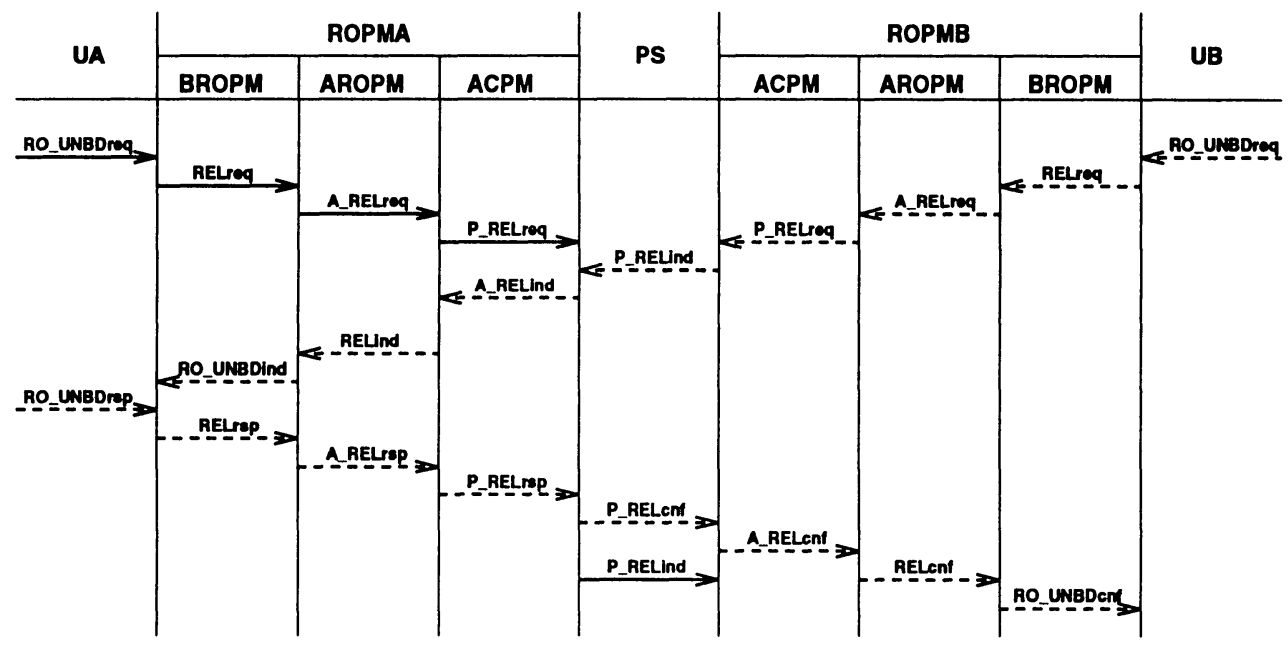

Figure 4. A sequence of service primitives causing Deadlock 7

our method described here provides a viable approach to verifying real ISO protocols specified in standard Estelle.

\section{REFERENCES}

1. ISO9074, Information Processing Systems - Open Systems Interconnection - ESTELLE (Formal Description Technique based on an Extended State Transition Model), 1989.

2. S. Budkowski and P. Dembinski, An Introduction to Estelle: A Specification Language for Distributed Systems, Computer Networks and ISDN Systems, Vol. 24, pages 3-23, 1987.

3. V. Dimitrov and A. Petkov, Verification Oriented Estelle Specification of Communication Protocols, R. Speth (ed.), Research into Networks and Distributed Applications, pages 953-960, North-Holland, 1988.

4. P. Azema, J.C. Lloret, G. Papapanagiotakis, and F. Vernadat, ESTELLE Validation and PROLOG Interpreted Petri Nets, M. Diaz, J.-P. Ansart, J.-P. Courtiat, P. Azema, and V. Chari (eds.), The Formal Description Technique Estelle, pages 273-302, NorthHolland, 1989.

5. J.P. Courtiat and P. de Saqui-Sannes, ESTIM: an integrated environment for the simulation and verification of OSI protocols specified in Estelle*, Computer Networks and ISDN Systems, Vol. 25, pages 83-98, 1992.

6. A. Jirachiefpattana and R. Lai, Verifying Estelle Specifications : Numerical Petri Nets Approach, 1993 International Conference on Network Protocols, pages 334-341, IEEE Computer Press, 1993. 
7. R. Lai and A. Jirachiefpattana, Verification of ISO ACSE protocol specified in Estelle, Computer Communications, Special issue on Upper Layer Protocols and Distributed Applications, Butterworth-Heinemann, March, 1994.

8. G.R. Wheeler, Numerical Petri Nets - A definition, Technical Report No. 7780, Telecom Australia, Research Laboratories 770 Blackburn Road, Clayton, Victoria, Australia $3168,1985$.

9. G.R. Wheeler, M.C. Wilbur-Ham, J. Billington, and J.A. Gilmour, Protocol Analysis Using Numerical Petri Nets, Lecture Notes in Computer Science, No. 188, pages 435$452,1986$.

10. J. Billington, G.R. Wheeler, and M.C. Wilbur-ham, PROTEAN: A High-level Petri Net Tool for the Specification and Verification of Communication Protocols, IEEE Transactions on Software Engineering, Vol. 14, No. 3, pages 301-316, March 1988.

11. F.J.W. Symons, Modelling and Analysis of Communication Protocols using Numerical Petri Nets, PhD thesis, Dep. Elec. Eng. Sci., Univ. Essex, Telecommun. Syst. Group Rep. 152, May 1978.

12. J. Billington, Specification of the Transport Service using Numerical Petri Nets, C. Sunshine (ed.), Second International Workshop on Protocol Specification, Testing and Verification, IFIP, Idyllwild, California, May, 1982.

13. R. Lai, T.S. Dillon, and K.R. Parker, Application of Numerical Petri Nets to Specify ISO FTAM Protocol, In Proceedings of the 1989 Singapore International Conference on Networks, July 19-20, 1989, Singapore.

14. ISO/IEC PDAM 9072-1-CCITT Rec. X.219, Remote Operations - Part 1: OSI Realisations - Remote Operations Service Element (ROSE) Service Definition, 1992.

15. ISO/IEC PDAM 9072-2-CCITT Rec. X.229, Remote Operations - Part 2: OSI Realisations - Remote Operations Service Element (ROSE) Protocol Specification, 1992.

16. R. Lai, T.S. Dillon, and K.R. Parker, Verification Results for ISO FTAM Basic Protocol, Ninth IFIP WG 6.1 International Symposium on Protocol Specification, Testing and Verification, 6th-9th June, The Netherlands, 1989.

17. S.T. Vuong and D.D. Cowan, Reachability Analysis of Protocols with FIFO Channels, ACM SIGCOM'83 Symposium on Communications Architectures and Protocols, pages 49-57, March, 1983.

18. R. Lai, K.R. Parker, and T.S. Dillon, On Using PROTEAN to Verify ISO FTAM Protocol, In Proceedings of the Workshop On Automatic Verification Methods For Finite State Systems, Grenoble, France, June 12-14, 1989. 


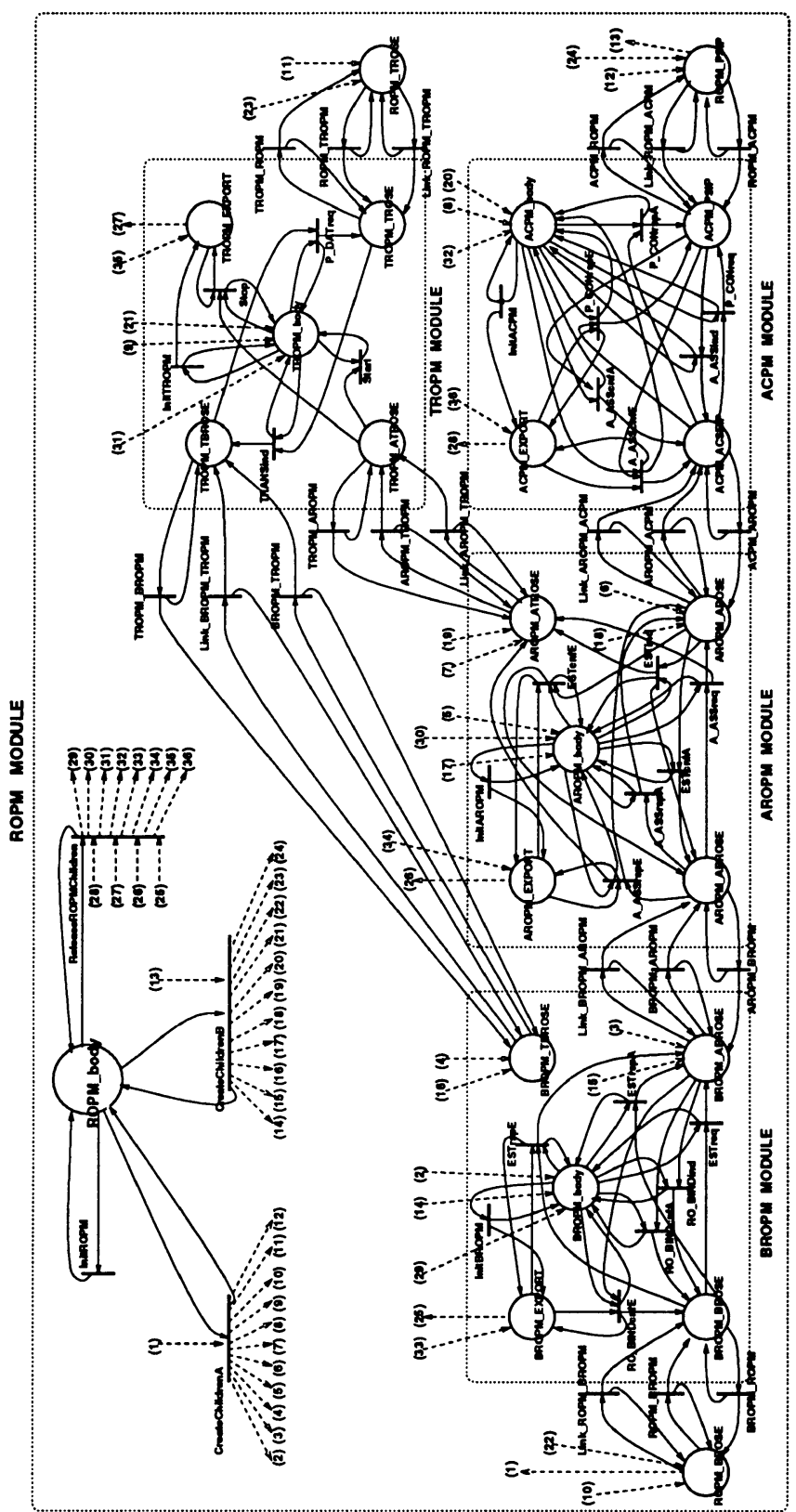

Figure 5. An NPN graph for the ROSE association establishment 


\section{Appendix A}

Estelle Specifications of ROSE Association Establishment module ROPM_type systemactivity; ip end

BROSE : BROPM_Channel_type (provider); PSIP : Pres_Connect_Channel_type (user); TROSE : Pres_Data_Channel_type (user);

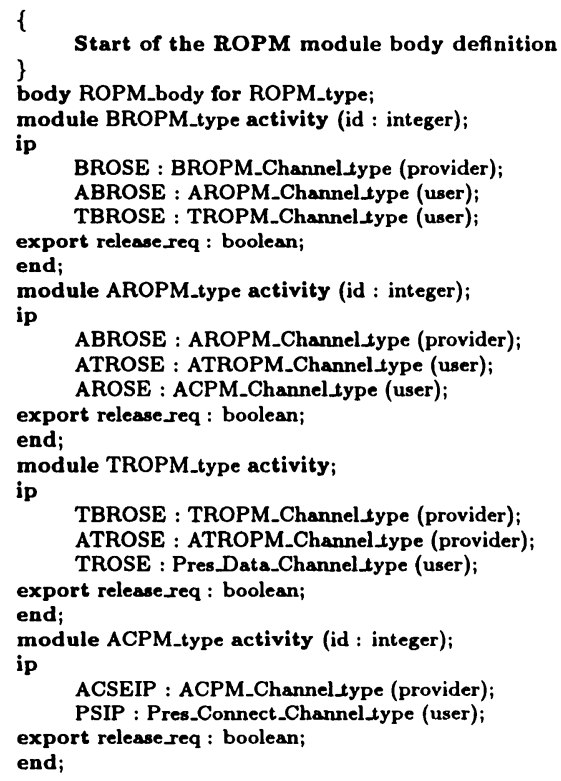

Start of the ROPM module body definition body ROPM_body for ROPM_type; module BROPM_type activity (id : integer); ip

BROSE : BROPM_Channel_type (provider);

ABROSE : AROPM_Channel type (user);

TBROSE : TROPM_Channel_type (user); export release_req : boolean;

end;

module AROPM_type activity (id : integer); ip

ABROSE : AROPM_Channel_type (provider);

ATROSE : ATROPM_Channel type (user)

AROSE : ACPM_Channel type (user);

export release_req : boolean;

end;

module TROPM_type activity;

ip

TBROSE : TROPM_Channel_ype (provider);

ATROSE : ATROPM_Channel type (provider);

TROSE : Pres_Data_Channel_type (user);

export release_req : boolean;

end;

module ACPM_type activity (id : integer);

ip

ACSEIP : ACPM_Channel_type (provider)

PSIP : Pres_Connect_Channel_type (user)

export release_req : boolean;

end; \}

Start of the BROPM module body definition body BROPM_body for BROPM_type; var

bad_number : integer;

roestrq : ESTRQ_TYPE;

roestrp : ESTRP_TYPE;

robindin : RO_BINDIN_TYPE;

robindcf : RO_BINDCF.TYPE;

state

STA01, (* unbound *)

STA02, (* bound *)

STA03A, (* local bind pending *)

STA03B, (* remote bind pending *)

STA04A, (* local unbind pending *)

STA04B, (* remote unbind pending *)

STA04C, (* unbind collision - responder completion *) initialize

STA04D; (* unbind collision - initiator completion *)

to STA01

begin

bad_number $:=0$;

releasedeq := FALSE; end;

trans

FROM STA01 TO STA03A

WHEN BROSE.RO_BINDreq

PROVIDED (id = 1)

NAME ESTreg:

begin

roestrq := event;

Output ABROSE.ESTreq (roestrq); end;

FROM STA01 TO STA03B

WHEN ABROSE.ESTind

PROVIDED (id $<>1$ )

NAME RO_BINDind:

begin

robindin := event

Output BROSE.RO_BINDind (robindin); end;

FROM STA03A TO STA02

WHEN ABROSE.ESTcnf

PROVIDED (id = 1) and (event.Outcome = RESULT) NAME RO_BINDcnfA:

begin

robindcf := event; end;

Output BROSE.RO_BINDcnf (robindcf);

FROM STA03A TO STA01

WHEN ABROSE.ESTcnf

PROVIDED (id = 1) and (event.Outcome = ERROR) NAME RO_BINDcnfE:

begin

robindcf := event;

Output BROSE.RO_BINDcnf (robindef);

end; release_req $:=$ TRUE

FROM STA03B TO STA02

WHEN BROSE.RO_BINDrsp

PROVIDED (id $<>$ 1) and (event.Outcome = RESULT)

NAME ESTrspA:

begin

roestrp := event; end;

Output ABROSE.ESTrsp (roestrp);

PROM STA03B TO STA01

WHEN BROSE.RO.BINDrap

PROVIDED (id $<>1$ ) and (event. Outcome = ERROR)

NAME ESTropE:

begin

roestrp : = event;

Output ABROSE.ESTrsp (roestrp);

end releasereq $:=$ TRUE

end; (* End of BROPM *)

\{ Start of the AROPM module body definition body AROPM_body for AROPM_type; var

aroestin : ESTIN.TYPE

aroestcf : ESTCF-TYPE

aroassrq : A_ASSRQ_TYPE;

aroassrp : A_ASSRP_TYPE; state

AA01, (* unassociated *)

AA02, (* associated *)

AA03, (* association pending *)

AA04, (* release pending ${ }^{*}$ )

AA05; (* release collision *) 
initialize

to AA01

begin
releasereq := FALSE;
end;

trans

FROM AA01 TO AA03

WHEN ABROSE.ESTreq

NAME A_ASS req:

begin

aroassrq.User Info := event;

Output AROSE.A_ASSreq (aroassrq)

end;

Output ATROSE.Start;

FROM AA01 TO AA03

WHEN AROSE.A_ASSind

NAME ESTind:

begin

aroestin := event.User Info

Output ABROSE.ESTind (aroestin); end;

Output ATROSE.Start:

FROM AA03 TO AA02

WHEN ABROSE.ESTrsP

PROVIDED (event. Outcome $=$ RESULT)

NAME A_ASSTrpA:

begin

aroassrp.Result := ACCEPTED;

aroassrp. User.Info := event;

Output AROSE.A_ASSrsp (aroassrp); end;

FROM AA03 TO AA01

WHEN ABROSE.ESTrsp

PROVIDED not (event.Outcome = RESULT)

NAME $A_{-}$ASS rsp E

begin

aroassrp.Result := REJECTED;

aroassrp. User Info := event;

Output AROSE.A_ASSrsp (aroassrp)

Output ATROSE.Stop

end;

release_req $:=$ TRUE;

FROM AA03 TO AA02

WHEN AROSE.A_ASScnf

PROVIDED (event.User Info.Outcome $=$ RESULT)

NAME ESTCnfA:

begin

aroestcf := event.User Info

Output ABROSE.ESTcnf (aroestcf); end;

FROM AA03 TO AA01

WHEN AROSE.A_ASScnf

PROVIDED not (event.User_Info.Outcome $=$ RESULT)

NAME ESTCnfE.

begin

aroestcf := event.User_Info;

Output ABROSE.ESTcnf (aroestcf);

Output ATROSE.Stop

end;

release_req := TRUE

end; (" End of AROPM *)

Start of the TROPM module body definition

body TROPM_body for TROPM_type;

var

trotrain : TRANSIN_TYPE;

trodatrq : TRANSRQ-TYPE state

TP01, (* inactive *)

initialize

TP02; (* active *)

to TP01

begin

end; release_req := FALSE;

trans

FROM TP01 TO TP02

WHEN ATROSE.Start

NAME Start:

begin

end;

FROM TP02 TO TP02

WHEN TBROSE.TRANSTeq

NAME P_DATreq:

begin

trodatrq := event

end;

Output TROSE.P_DATreq(trodatrq);

FROM TP02 TO TP02

WHEN TROSE.P.DATind

NAME TRANSind:

begin

trotrain := event; end;

Output TBROSE.TRANSind(trotrain);

FROM TP02 TO TP01

WHEN ATROSE.Stop

NAME Stop:

begin

end

release seq := TRUE

end; (* End of TROPM *)

\{

Start of the ACPM module body definition body ACPM_body for ACPM_type;

var

pconrq : P_CONRQ_TYPE;

pconrp : P.CONRP_TYPE

aassin : A_ASSIN_TYPE

state aasscf : A_ASSCF.TYPE

STA0, (* idle *)

STA1, (* awaiting AARE *)

STA2, (* awaiting A-ASCrsp *)

STA3, (* awaiting RLRE *)

STA4, (* awaiting A-RLSrsp *)

STA5, (* associated *)

STA6, (* collision assoc init *)

initialize

STA7; (* collision assoc resp *)

to STAO

begin

end

release_req $:=$ FALSE;

trans

FROM STAO TO STA1

WHEN ACSEIP.A_ASSreq

NAME P_CONreq:

begin

pconrq := event: end;

Output PSIP.P_CONreq (pconrq);

FROM STAO TO STA2

WHEN PSIP.P_CONind 
NAME A_ASSind: begin aassin := event; end;

Output ACSEIP.A_ASSind (assin);

FROM STA2 TO STA5

WHEN ACSEIP.A_ASSrsp

PROVIDED (event.Result $=$ ACCEPTED)

NAME P_CONrspA:

begin

pconrp.Result := P_ACCEPTANCE;

pconrp.User_Data.Result : = event.Result;

end;

Output PSIP.P_CONrsp (pconrp)

FROM STA2 TO STAO

WHEN ACSEIP.A_ASSrsp

PROVIDED not (evist. Result = ACCEPTED)

NAME P_CONrspE:

begin

pconrp.Result := USER_REJECTION;

pconrp.User_Data.Result := event.Result;

Output PSIP.P_CONrsp (pconrp); end;

release_req := TRUE;

FROM STA1 TO STA5

WHEN PSIP.P_CONenf

PROVIDED (event. Result = P_ACCEPTANCE)

NAME $A_{-} A S S c n f A$ :

begin

assscf.Result := event.User-Data.Result ; end;

Output ACSEIP.A_ASScnf (aasscf)

FROM STA1 TO STAO

WHEN PSIP.P_CONenf

PROVIDED not (event.Result $=$ P_ACCEPTANCE)

NAME $A \_A S S c n f E$ :

begin

aasscf.Result := event.User Data.Result;

Output ACSEIP.A_ASScnf (aasscf);

end;

release_req := TRUE;

end; ( ${ }^{*}$ End of ACPM *)

\}

Main body of ROPM module

AROPM_FREE, BROPM_FREE,

ACPM_FREE, TROPM_FREE : boolean;

(* Module Variable Declaration Part *)

modvar

AROPM : AROPM-type;

BROPM : BROPM-type;

TROPM : TROPM-type;

initialize

ACPM : ACPM_type;

begin

AROPM_FREE := TRUE;

BROPM_FREE $:=$ TRUE;

ACPM_FREE $:=$ TRUE;

end;

TROPM_FREE $:=$ TRUE;

trans

WHEN BROSE.RO_BINDreq

PROVIDED AROPM_FREE and BROPM_FREE and ACPM_FREE and TROPM_FREE

NAME CreateChildrenA:

begin

init AROPM with AROPM_body(1); init BROPM with BROPM_body(1);

init ACPM with ACSE_body(1);

init TROPM with TROPM_body;

connect BROPM.ABROSE to AROPM.ABROSE connect AROPM.AROSE to ACPM.ACSEIP; connect BROPM.TBROSE to TROPM.TBROSE; connect AROPM.ATROSE to TROPM.ATROSE; attach BROSE to BROPM.BROSE;

attach PSIP to ACPM.PSIP;

attach TROSE to TROPM.TROSE;

AROPM_FREE := FALSE

BROPM_FREE $:=$ FALSE;

ACPM_FREE := FALSE;

TROPM_FREE $:=$ FALSE; end;

trans

WHEN PSIP.P_CONind

PROVIDED AROPM_FREE and BROPM_FREE and

ACPM_FREE and TROPM.FREE

NAME CreateChildrenB:

begin

init AROPM with AROPM_body(2)

init BROPM with BROPM_body(2);

init ACPM with ACSE_body(2);

init TROPM with TROPM_body;

connect BROPM.ABROSE to AROPM.ABROSE

connect AROPM.AROSE to ACPM.ACSEIP;

connect BROPM.TBROSE to TROPM.TBROSE;

connect AROPM.ATROSE to TROPM.ATROSE

attach BROSE to BROPM.BROSE;

at tach PSIP to ACPM.PSIP;

at ach TROSE to TROPM.TROSE;

AROPM_FREE := FALSE;

BROPM_FREE $:=$ FALSE;

ACPM_FREE $:=$ FALSE;

TROPM_FREE := FALSE;

rans end;

PROVIDED exist X1:BROPM_type suchthat X1.release_req NAME ReleaseROPMChildren:

begin

forone X1:BROPM_type suchthat X1.releasereq do forone X2:AROPM_type suchthat X2.releasereq do forone X3:TROPM_type suchthat X3.release_req do forone X4:ACPM_type suchthat X4.release_req do begin

release $\mathrm{X1}$;

release $\mathrm{X}$;

release X3;

end,

release $\mathrm{X4}$;

end;

end; (* End of ROPM *) 